\title{
A UTILIZAÇÃO DA PESQUISA OPERACIONAL PARA RESOLVER PROBLEMA DE LOGÍSTICA EM TRANSPORTE RODOVIÁRIO DE CARGAS
}

The employment of Operations Research to solve logistics problem in road freight transport

\author{
Marcus Ayrton Rocha de Lima ${ }^{1}$; Douglas Fernandes ${ }^{1}$; Flavio Alberto \\ Oliva $^{2}$
}

\author{
${ }^{1}$ Faculdade de Tecnologia de Presidente Prudente \\ E-mail: marcus.lima5@fatec.sp.gov.br \\ ${ }^{2}$ Universidade do Oeste Paulista - UNOESTE, Presidente Prudente, SP \\ E-mail: flavio.oliva@unoeste.br
}

RESUMO - O transporte rodoviário de cargas é um segmento dinâmico, complexo e competitivo, o qual vai demandar de seus gestores decisões rápidas e acertadas, pois o simples fato de disponibilizar um veículo na estrada ou cidade para atender às necessidades dos clientes não implica em geração de resultados positivos. Assim, as tomadas de decisões neste setor deverão ser minuciosamente estudadas para a sobrevivência dos negócios. O cenário para este estudo é uma empresa de transportes rodoviários de cargas fictícia que, por meio de um de seus veículos, deverá atender a dois clientes específicos. O objetivo deste artigo é demonstrar a utilização da Pesquisa Operacional (PO) como suporte nas tomadas de decisões mais complexas, tendo como subsídio o recurso Solver disponível em planilhas Microsoft Excel, vislumbrando solucionar um problema de logística e simulações de resultado de uma empresa prestadora de serviços em transporte, baseando-se em restrições inerentes ao empreendimento em que a função objetivo deverá encontrar o ponto de equilíbrio e a maximização do resultado.

Palavras-chave: Simulação; Maximização; Transportes.

Recebido em: 08/08/2018 Revisado em: $11 / 08 / 2018$ Aprovado em: 18/09/2018
ABSTRACT - Road freight transport is a dynamic, complex and competitive segment that will require quick and accurate decisions from its managers, since the mere fact of making a vehicle available on the road or in the city to meet customer needs does not imply the generation of results positive. Thus, decision-making in this sector should be carefully studied for the survival of the business. The scenario for this study is a fictitious cargo road transport company that, through one of its vehicles, should serve two specific customers. The aim of this article is to demonstrate the employment of Operations Research (OR) as a support in the most complex decision making, based on the Solver resource available in Microsoft Excel spreadsheets, aiming to solve a logistics problem and result simulations of a company that provides 
transport services, based on constraints inherent to the enterprise in which the objective function must find the breakeven point and maximize the result.

Keywords: Simulation; Optimization; Transportation. 


\section{INTRODUÇÃO}

A todo momento, nos ambientes organizacionais, seus membros tomarão decisões, indo daquelas mais simples às mais complexas, rotineiras ou desestruturadas, erradas ou acertadas, com muita ou pouca informação. De forma simples e objetiva, a tomada de decisão é a escolha entre alternativas para resolver problemas ou aproveitar oportunidades. Para Maximiano (2009, p.58) decisões são tomadas para resolver problemas ou aproveitar oportunidades.

No ano de 1947, com o propósito de explicar o comportamento humano nas organizações, Herbert Simon escreve a obra Comportamento Administrativo, dando início à Teoria das Decisões, onde “... cada pessoa participa racional e conscientemente, escolhendo e tomando decisões individuais a respeito de alternativas racionais de comportamento." (CHIAVENATO, 2004, p.348).

A organização é um sistema de decisões em que cada pessoa participa consciente

racionalmente,

escolhendo e decidindo entre alternativas mais ou menos racionais que são apresentadas de acordo com sua personalidade, motivações e atitudes. Os processos de percepção das situações e o raciocínio são básicos para a explicação do comportamento humano nas organizações: o que uma pessoa aprecia e deseja influencia o que se vê e interpreta, assim como o que vê e interpreta influencia o que aprecia e deseja. Em outros termos, a pessoa decide em função de sua percepção das situações. Em resumo, as pessoas são processadores de informação, criadoras de opinião e tomadoras de decisão. CHIAVENATO 2004, p.348).

O processo de tomada de decisões pode ser complexo, pois em muitos casos depende de características individuais do tomador de decisões, as situações em que está envolvido, sejam pessoais ou profissionais e sua maneira de perceber e intuir as situações que se apresentam. As decisões podem ser influenciadas por diversos fatores como: racionalidade, personalidade, percepção e intuição. As tomadas de decisão em logística são praticamente, em sua totalidade, racionais.

A diferença entre racionalidade e intuição está na proporção de informação, de um lado, e opinião e sentimentos, de outro. Quanto maior a base de informação, mais racional é o processo. Quanto maior a proporção de opiniões e sentimentos, mais intuitivo se torna. A racionalidade e a intuição são atributos humanos complementares e não concorrentes.

(MAXIMIANO, 2009, p.71)

Para Chiavenato (2004, p.348), toda decisão envolve seis elementos a saber:

1) O tomador de decisão: é a pessoa que faz uma escolha ou opção entre várias alternativas futuras de ação.

2) Os objetivos: são o que o tomador de decisão pretende alcançar com suas ações.

3) As preferências: são os critérios que o tomador de decisão usa para fazer sua escolha.

4) A estratégia: é o curso de ação que o tomador de decisão escolhe para atingir seus objetivos dependendo dos recursos que pode dispor.

5) A situação: são os aspectos do ambiente que envolve o tomador de decisão, alguns deles fora do seu controle, conhecimento ou compreensão e que afetam sua escolha.

6) O resultado: é a consequência ou resultado de uma estratégia. 
Outra variável importante a ser considerada nas tomadas de decisão é o tempo disponível para sua efetivação. Como em qualquer outro setor, o tempo é escasso também para os transportadores de cargas e para seus gestores, promovendo a probabilidade em se tomar decisões equivocadas dada a pressa. Uma decisão de fazer ou não um frete, deve ser acertada, pois além da possibilidade de se gerar prejuízo, há muita concorrência no segmento. $O$ número de operadores autônomos concorrendo com empresas estabelecidas é muito grande. Em 2016, segundo dados da Agência Nacional de Transportes Terrestres (ANTT), havia 622.328 transportadores autônomos registrados contra 161.264 empresas de transportes de cargas formalizadas.

A qualidade das informações que se dispõe para o auxílio à modelagem matemática é um problema a ser considerado. O desconhecimento de uma boa parte dos transportadores no que diz respeito ao detalhamento e estudo de seus custos é outro fator a ser ressaltado em meio às decisões a serem tomadas. Os custos são considerados informações estruturadas e a base da tomada de decisões são informações. Sem a riqueza de tais contribuições, as decisões, muito provavelmente, serão mal tomadas e prejudicadas.

Não se pode prescindir da utilização da intuição nas decisões a serem tomadas. Os gestores, ao se depararem com alternativas conflitantes e concorrentes, podem realizar modelagens e simulações, mas também devem utilizar-se de suas experiências profissionais e seus pressentimentos, caso contrário, um suporte importante, que é o conhecimento humano, estará sendo desperdiçado. Conforme Lachtermacher (2009, p.3), "muitos gerentes deixaram de utilizar sua intuição completamente, o que é bastante prejudicial ao processo de tomada de decisão, pois uma base de conhecimentos pode estar sendo desperdiçada". O objetivo deste trabalho é demonstrar a utilização da Pesquisa Operacional para subsidiar os gestores nas tomadas de decisão em transporte rodoviário de cargas utilizando a ferramenta Solver. Em primeiro plano, o modelo deverá simular o alcance do ponto de equilíbrio do veículo e em segundo lugar a maximização do resultado otimizando o equipamento.

\section{METODOLOGIA}

O conhecimento relativo à gestão de negócios, para a prototipação desejada em trabalhos dessa natureza, pode propiciar ganhos de tempo ao processo de modelagem. Foram utilizados entendimentos de contabilidade, custos, finanças e alguns dados relativos ao veículo fictício proposto no estudo foram pesquisados. A construção da modelagem matemática inicia-se pela adoção de uma notação apropriada para as principais quantidades presentes na definição do problema, denotando-se por $x_{1}$, $x_{2} \ldots x_{n}$ as quantidades manipuladas do problema. Atribui-se o nome de variáveis de decisão a estas quantidades. O problema é redefinido por meio de fórmulas, relações matemáticas ou proposições. Uma fórmula denominada de função é utilizada para descrever como o objetivo do problema é influenciado pelos valores das variáveis de decisão. As relações matemáticas envolvendo os símbolos "=", "<", ">" e proposições gerais são empregadas para descrever eventuais restrições para a escolha de valores para as variáveis de decisão. A prescrição significa otimizar a função objetivo sujeito às restrições sendo que, otimizar pode significar minimizar ou maximizar, ou seja, determinar os valores das variáveis de decisão que conduzem ao menor ou maior valor para a função objetivo.

A validação do modelo ocorre por meio da verificação da compatibilidade entre a solução encontrada e a realidade do problema. As características relevantes do problema devem estar presentes na modelagem, dessa forma a solução encontrada é implementável. Caso contrário, 
um novo ciclo de modelagem e obtenção de solução deverá ser desenvolvido. O processo se faz por meio da modelagem na ferramenta Solver, disponível no pacote de suplementos do Microsoft Excel.

\section{RESULTADOS}

Este estudo parte da criação fictícia de uma empresa prestadora de serviços de transporte rodoviário de cargas. A empresa em tela possui vários clientes e entre estes, dois clientes específicos que têm contrato de fornecimento mensal de cargas com a empresa que deverão ser cumpridos. 0 modelo deverá apresentar como objetivo as quantidades a serem transportadas de cada produto para cada cliente devendo, em primeiro plano, maximizar o resultado da empresa (maior Lucro Líquido possível). Em um segundo momento o modelo deverá sugerir as quantidades a serem transportadas que atendam aos critérios contratuais e proporcionem $\mathrm{o}$ alcance do ponto de equilíbrio. A empresa, para atender esses dois clientes citados, disponibiliza um veículo trucado com as características conforme o quadro 1 a seguir.

Quadro 1. Características do veículo

\begin{tabular}{|c|c|c|}
\hline CAMINHÃO TRUCADO & 12.500 & $\mathrm{Kg}$ \\
\hline \multicolumn{3}{|c|}{ BAÚ - CUBAGEM } \\
\hline ALTURA & 2,50 & metros \\
\hline LARGURA & 2,50 & metros \\
\hline COMPRIMENTO & 7,50 & metros \\
\hline CUBAGEM & 46,875 & $\mathrm{~m}^{3}$ \\
\hline
\end{tabular}

Conforme os dados do quadro 1 , temos como as duas primeiras restrições à resolução do problema, a capacidade de transporte de cargas do veículo limitada a $12.500 \mathrm{~kg}$, como também em metros cúbicos do baú, com sua limitação em $46,875 \mathrm{~m}^{3}$.
Quadro 2. Tipos de cargas disponíveis

\begin{tabular}{|l|r|l|l|l|}
\hline \multicolumn{4}{|c|}{ DOIS TIPOS DECARGASDISPONIVEIS } \\
\hline CARACTERISTICAS & CLLENTE1 & UNID.MEDIDA & CLIENTE2 & UNID. MEDIDA \\
\hline QUANTIDADEDE ITENS/MÊS & 150 & Unidades & 3.800 & Unidades \\
\hline PESO UNITÁRIO & 45,00 & $\mathrm{Kg}$ & $4,125 \mathrm{Kg}$ \\
\hline PESO TOTAL & 6.750 & $\mathrm{Kg}$ & $15.675 \mathrm{Kg}$ \\
\hline ATENDIMENTOCONTRATO & $40,00 \%$ & & $30,00 \%$ \\
\hline QUANTIDADES MÍNIMAS & 60 & Unidades & 1.140 & Unidades \\
\hline
\end{tabular}

De acordo com o quadro 2, os dois clientes estudados disponibilizam produtos de sua fabricação com as características apresentadas. Há a necessidade de atender, conforme contrato, um mínimo de unidades mensais para cada cliente.

Quadro 3. Características individuais das cargas

\begin{tabular}{|c|c|c|c|c|}
\hline \multicolumn{5}{|c|}{ CUBAGEM POR TTEM } \\
\hline VARIÁVEESS & CLIENTE1 & UNID.ME & CLIENTE2 & UNID.MEDIDA \\
\hline ALTURA & 0,775 & Metros & 0,138 & Metros \\
\hline LARGURA & 0,808 & Metros & 0,158 & Metros \\
\hline PROFUNDIDADE & 0,690 & Metros & 0,223 & Metros \\
\hline CUBAGEM UNITÁRIO & 0,432 & $M^{3}$ & 0,005 & $M^{3}$ \\
\hline CUBAGEMTOTAL & 64,812 & $M^{3}$ & 76,155 & $M^{3}$ \\
\hline PESOCUBADO & $19.357,09$ & $K G$ & $22.744,96$ & $K G$ \\
\hline
\end{tabular}

Os dados do quadro 3 apresentam as características individuais dos produtos disponibilizados para a prestação do serviço, como cubagem (Altura $X$ Largura $X$ Profundidade), a cubagem total de cada item (multiplicação dos itens disponibilizados pela cubagem unitária). Percebe-se que o atendimento dos dois clientes fica prejudicado se o for realizado em sua totalidade, já que o Cliente 1 , em seu produto, ocuparia $64,812 \mathrm{~m}^{3}$ do baú que tem capacidade de $46,875 \mathrm{~m}^{3}$ como visto anteriormente. Já o atendimento ao Cliente 2 , que tem sua carga total cubada em 76,155 $\mathrm{m}^{3}$, mais prejudicado ainda. 
Quadro 4. Distância a ser percorrida para o atendimento ao serviço

\begin{tabular}{|l|r|r|r|}
\hline \multicolumn{3}{|c|}{ DISTÂNCIA PERCORRIDA PARA ATENDER À DEMANDA } \\
\hline & CLIENTE 1 & & CLIENTE 2 \\
\cline { 1 - 2 } & 2.000 & & 1.500 \\
\hline
\end{tabular}

No quadro número 4 é especificada a distância em quilômetros, percurso ida e volta, para atender aos serviços solicitados, ou seja, um total de $3.500 \mathrm{~km}$. Os dois clientes estão na mesma rota estabelecida pela empresa.

A empresa fictícia está enquadrada no regime de tributação Lucro Presumido com uma alíquota de $17,93 \%$ e paga comissão sobre serviço ao motorista de $10 \%$

Outros fatores importantes, que irão influenciar a tomada de decisão, são os custos variáveis de $\mathrm{R} \$ 2,54$ por km rodado, os custos fixos mensais do veículo simulado que somam $\mathrm{R} \$ 10.800,00$, bem como os preços praticados por $\mathrm{kg} / \mathrm{cubado}$ sendo: Para $\mathrm{o}$ Cliente 1 , segundo o contrato firmado, cada Kg cubado gera receita de $\mathrm{R} \$ 2,98$ e para o Cliente 2, conforme o contrato, o valor a ser cobrado por kg cubado é de $\mathrm{R} \$ 3,22$.

Quadro 5. Margem de Contribuição Total (MCT) por Cliente para atender o ponto de equilíbrio

\begin{tabular}{|l|r|r|r|}
\hline \multicolumn{3}{|c|}{ DEMONSTRAÇÃO DO RESULTADO DO PONTO DE EQUILÍBRIO } \\
\hline VARIÁVEIS/CLIENTES & \multicolumn{1}{c|}{ CLIENTE1 } & \multicolumn{1}{c|}{ CLIENTE2 } & \multicolumn{1}{c|}{ TOTAL } \\
\hline QUANTIDADE DE ITENS & 60 & 1.611 & \\
\hline CUBAGEM & 25,9247 & 7,8251 & 33,7498 \\
\hline PESO (Kg) & $2.700,00$ & $6.643,93$ & $9.343,93$ \\
\hline PESO CUBADO & $6.913,25$ & $2.086,70$ & $8.999,95$ \\
\hline RECEITA BRUTA & $20.601,48$ & $6.719,18$ & $27.320,66$ \\
\hline$(-)$ CUSTO VARIÁVEL & $5.080,00$ & $3.810,00$ & $8.890,00$ \\
\hline$(-)$ IMPOSTOS & $3.693,85$ & $1.204,75$ & $4.898,59$ \\
\hline$(-)$ COMISSÕES & $2.060,15$ & 671,92 & $2.732,07$ \\
\hline MCT & $9.767,49$ & $1.032,51$ & $10.800,00$ \\
\hline CUSTOS FIXOS ==============================> & $10.800,00$ \\
\hline RESULTADO OPERACIONAL ====================> & - \\
\hline
\end{tabular}

Ao verificar o os resultados do quadro 5, nota-se que ao atender em $40 \%$ à demanda do Cliente 1 , que é a quantidade mínima a ser transportada, conforme a restrição contratual de atendimento à disponibilidade de produtos do cliente, transportando 60 das 150 unidades disponíveis e $42,39 \%$ de grau de atendimento ao Cliente 2, em 1.611 unidades das 3.800 disponíveis, alcança-se o ponto de equilíbrio. Neste ponto de operação, o veículo é utilizado em sua capacidade cúbica com $72,00 \%$, ou seja, $33,7498 \mathrm{~m}^{3}$ de um total disponível de 46,875 $\mathrm{m}^{3}$ e na sua capacidade de peso em $74,75 \%$, com $9.343,93 \mathrm{~kg}$ de um total possível de $12.500 \mathrm{~kg}$.

Quadro 6. Margem de Contribuição Total (MCT) por Cliente para a maximização do frete

\begin{tabular}{|l|r|r|r|}
\hline \multicolumn{4}{|c|}{ DEMONSTRAÇÃO DO RESULTADO DA MAXIMIZAÇÃO } \\
\hline VARIÁVEIS/CLIENTES & \multicolumn{1}{c|}{ CLIENTE1 } & \multicolumn{1}{c|}{ CLIENTE2 } & \multicolumn{1}{c|}{ TOTAL } \\
\hline QUANTIDADE DE ITENS & 85 & 2.105 & \\
\hline CUBAGEM & 36,6481 & 10,2269 & 46,8750 \\
\hline PESO (Kg) & $3.816,82$ & $8.683,18$ & $12.500,00$ \\
\hline PESO CUBADO & $9.772,81$ & $2.727,19$ & $12.500,00$ \\
\hline RECEITA BRUTA & $29.122,99$ & $8.781,54$ & $37.904,52$ \\
\hline$(-)$ CUSTO VARIÁVEL & $5.080,00$ & $3.810,00$ & $8.890,00$ \\
\hline$(-)$ IMPOSTOS & $5.221,75$ & $1.574,53$ & $6.796,28$ \\
\hline$(-)$ COMISSÕES & $2.912,30$ & 878,15 & $3.790,45$ \\
\hline MCT & $15.908,94$ & $2.518,85$ & $18.427,79$ \\
\hline CUSTOS FIXOS =============================> & $10.800,00$ \\
\hline RESULTADO OPERACIONAL ==================== & $7.627,79$ \\
\hline
\end{tabular}

O modelo agora demonstrado e as simulações executadas tiveram o objetivo de maximização de resultados para a empresa, ou seja, o maior resultado possível após o pagamento dos custos fixos do veículo que somam, mensalmente, $\mathrm{R} \$ 10.800,00$, conforme observa-se no quadro 6. Nesta simulação o atendimento ao Cliente 1 é de $56,55 \%$, o que resulta em 85 unidades das 150 disponíveis do contrato e ao Cliente 2 é de $55,40 \%$, ou seja, 2.105 unidades das 3.800 disponibilizadas. Nota-se que esta situação é que atende aos limites de cubagem do veículo, e sua capacidade de carga em peso, $12.500 \mathrm{~kg}$ e também em $100 \%$ o limite da cubagem de $46,875 \mathrm{~m}^{3}$ resultando em um lucro de $\mathrm{R} \$ 7.627,79$.

\section{DISCUSSÃO}

A Pesquisa Operacional diz respeito à criação de modelos matemáticos para a resolução de problemas complexos em empresas. O termo foi utilizado, pela 
primeira vez, em 1938, visando estudar problemas de estratégia e tática (logística) decorrente de operações militares.

o desenvolvimento metodológico mais importante do período pós-guerra foi o Método Simplex, criado por George Dantzig, em 1947, para a resolução de problemas de Programação Linear, isto é, de problemas de planejamento nos quais são utilizados modelos de otimização lineares. Além disto a proliferação dos microcomputadores aliada ao aumento da velocidade de processamento dos mesmos fez com que os estudos de P.O. prosperassem.

Os estudos de P.O. no Brasil tiveram início na década de 1960, quando houve a realização do primeiro Simpósio Brasileiro de Pesquisa Operacional (SBPO), em 1968, no ITA - Instituto Tecnológico da Aeronáutica . Em seguida foi criada a Sociedade Brasileira de Pesquisa Operacional (SOBRAPO).

A empresa americana Frontline Systems Inc. adquiriu os direitos de utilização do método GRG (Gradiente Reduzido Generalizado), criado em 1966 por Carpentier e Abadie e, a partir desse método criou o Solver em diferentes versões que podiam ser adquiridas por todos que desejassem. Empresas como NASA, Bell Aerosystems, Boeing ou empresas de menor porte que eram atendidas pela Frontline de forma diferenciada, para que comprassem seus produtos. A Frontline iniciou uma parceria com a Microsoft, na década de 1980, trabalhando em versões anteriores do Windows e do Excel. Foi desenvolvida uma versão específica do Solver para uso através de planilhas.

\section{CONSIDERAÇÕES FINAIS}

Um dos grandes desafios dos gestores da área de transporte rodoviário de cargas é a acurácia do levantamento de custos dos veículos, pois a qualidade da informação deverá determinar o nível das tomadas de decisão. O tomador de decisões, a partir de um modelo envolvendo os conceitos de pesquisa operacional, juntamente com uma ferramenta de relativa facilidade de utilização, como é o caso do Solver presente no Microsoft Excel, pode ter sua ação extremamente facilitada. A modelagem aqui desenvolvida teve os objetivos de determinar o ponto de equilíbrio e maximizar o resultado da empresa. Outrossim, a rapidez das tomadas de decisão, fator que o mundo atual exige dos gestores, com a possibilidade de grau de acerto maior, é outra vantagem da utilização da pesquisa operacional em modelagem no Solver, além do fato que a ferramenta Solver está disponível no pacote da Microsoft Excel, ou seja, de fácil acesso.

A empresa, pelo fato de poder atender o cliente em suas demandas, e em sete dias de utilização do equipamento, cobrindo seus custos fixos, poderá trabalhar com tranquilidade, pelo menos no que diz respeito a essa rota. $O$ modelo não contabilizou a possibilidade de fretes retorno, o que ajudaria a pagar os custos fixos com mais facilidade.

\section{REFERÊNCIAS}

CHIAVENATO, I. Introdução à teoria geral da administração. 7. ed. Rio de Janeiro: Campus, 2004. 630 p.

LACHTERMACHER, Gerson. Pesquisa operacional na tomada de decisões. 4. ed. São Paulo: Pearson - Prentice Hall, 2009. 223 p.

MAXIMIANO, A. C. A. Introdução à Administração. Ed. Compacta. São Paulo: Atlas, 2009. 294p.

AGÊNCIA NACIONAL DE TRANSPORTES TERRESTRES (ANTT). Disponível em: http://www.antt.gov.br/cargas/arquivos old Estatisticas.html. Acesso: 01 ago. 2018.

MICROSOFT SOLVER EXCEL. Disponível em: https://support.office.com/ptbr/article/In\%C3\%ADcio-r\%C3\%A1pidoativar-e-usar-um-suplemento-2df59054-a46f- 
4fe4-bcb5-92ea461c315f. Acesso: 20 jun.

2018.

SOBRAPO - Sociedade Brasileira de Pesquisa

Operacional. Disponível em: <

http://www.sobrapo.org.br/o-que-e-

pesquisa-operacional>. Acesso: 07 Julho

2018. 